\title{
Upper limit for the accelerating gradient in the collinear wakefield accelerator as a function of the transformer ratio
}

\author{
S. S. Baturin* \\ The University of Chicago, PSD Enrico Fermi Institute, 5640 S Ellis Ave, Chicago, Illinois 60637, USA
}

\section{A. Zholents ${ }^{\dagger}$}

Argonne National Laboratory, Lemont, Illinois 60439, USA

(Received 27 March 2017; published 19 June 2017)

\begin{abstract}
The interrelation between the accelerating gradient and the transformer ratio in the collinear wakefield accelerator has been analyzed. It has been shown that the high transformer ratio and the high efficiency of the energy transfer from the drive bunch to the witness bunch can only be achieved at the expense of the accelerating gradient. Rigorous proof is given that in best cases of meticulously shaped charge density distributions in the drive bunch, the maximum accelerating gradient falls proportionally to the gain in the transformer ratio. Conclusions are verified using several representative examples.
\end{abstract}

DOI: 10.1103/PhysRevAccelBeams.20.061302

\section{INTRODUCTION}

In the collinear wakefield accelerator (CWA) ([1-8]) the drive electron bunch generates the electromagnetic field by interacting with the surrounding environment, typically formed either by the dielectric lined waveguide or the waveguide with small corrugations or plasma medium. This field, known as the wakefield, accelerates electrons of the witness bunch located at a strategically chosen distance behind the drive bunch with the maximum accelerating field and decelerates electrons of the drive bunch. The charge of a witness bunch is much smaller then the charge of a drive bunch. For the CWA to be an efficient accelerator, the maximum accelerating field must be much higher than the maximum decelerating field, in which case the energy gain experienced by witness electrons by the time the drive bunch is completely depleted of its energy will be much higher than the initial energy of the drive bunch electrons. This is accomplished by using the drive bunch with an asymmetric electron distribution along the bunch length [9]. In this paper we give a rigorous proof (in a full agreement with $[9,10]$ ) that the highest ratio of the maximum accelerating field to the maximum decelerating field is obtained using the drive bunch electron distribution that produces an identical decelerating field for all electrons inside the drive bunch. Expanding on this result, we also show that for a given environment and a charge of the drive bunch, the upper value of the accelerating field is strictly

\footnotetext{
s.s.baturin@gmail.com
}

azholents@aps.anl.gov

Published by the American Physical Society under the terms of the Creative Commons Attribution 4.0 International license. Further distribution of this work must maintain attribution to the author(s) and the published article's title, journal citation, and DOI. bounded by the ratio of the maximum accelerating field to the maximum decelerating field. This has always been observed in numerical simulations (and most recently reported in $[11,12])$, but to our knowledge has never been shown in the form of a theorem for arbitrary longitudinal bunch distribution as it is demonstrated in this paper. The results obtained in this paper are applicable to the CWA employing a single drive bunch. The CWA with a specially arranged train of drive bunches was examined analytically in Ref. [13].

The paper is organized in the following way. We obtain main results in the first two sections analyzing a generic CWA not limited to any specific symmetry either cylindrical or planar and to any specific type of the wakefield inducing environment, but limited only to the cases with a single mode wakefield Green's function. After that we prove that the collinear wakefield accelerators with the multimode Green's function cannot have higher accelerating gradient than that in the CWA with the single mode Green's function. Finally, we demonstrate a good agreement of the results with numerical calculations applied to several representative examples using dielectric lined CWA.

\section{TRANSFORMER RATIO}

We define charge distribution in the electron bunch as $q(s)$ and consider bunches localized on the interval $0 \leq s \leq l$. Therefore, we have

$$
\int_{0}^{l} q(s) d s=Q
$$

where $Q$ is the total bunch charge. Using the Green's function $G(s)$ consisting only of a fundamental harmonic (a multimode case will be considered later) $G(s)=$ $2 \kappa_{\|} \cos (k s) \theta(s)$, where $\kappa_{\|}$is the loss factor of a point 
particle per unit length, $k=2 \pi / \lambda$ is the wave vector, $\lambda$ is the wavelength, and $\theta$ is the Heaviside theta-function, we write for the longitudinal field inside of the electron bunch $[14,15]$

$E_{-}(s)=2 \kappa_{\|} \int_{0}^{s} \cos \left[k\left(s-s_{0}\right)\right] q\left(s_{0}\right) d s_{0}, \quad s \leq l$

and for the longitudinal field behind the electron bunch

$E_{+}(s)=2 \kappa_{\|} \int_{0}^{l} \cos \left[k\left(s-s_{0}\right)\right] q\left(s_{0}\right) d s_{0}, \quad s>l$.

Equation (2) is a Volterra equation of the first kind for the function $q$ and known $E_{-}$with the trigonometric kernel $\cos \left[k\left(s-s_{0}\right)\right]$. If we assume that $E_{-}(0)=0$ at the bunch "head", then the solution of Eq. (2) is [16]

$$
q(s)=\frac{1}{2 \kappa_{\|}}\left[E_{-}^{\prime}(s)+k^{2} \int_{0}^{s} E_{-}(x) d x\right],
$$

where the derivative is taken over $s$. Now one can express $E_{+}(s)$ as a function of $E_{-}(s)$ by substituting Eq. (4) into Eq. (3)

$$
\begin{aligned}
E_{+}(s)= & \int_{0}^{l} E_{-}^{\prime}\left(s_{0}\right) \cos \left[k\left(s-s_{0}\right)\right] d s_{0} \\
& +k^{2} \int_{0}^{l} \cos \left[k\left(s-s_{0}\right)\right] d s_{0} \int_{0}^{s_{0}} E_{-}(x) d x .
\end{aligned}
$$

Evaluating the first integral in Eq. (5) by parts we arrive at

$$
\begin{aligned}
E_{+}(s)= & E_{-}(l) \cos [k(s-l)] \\
& -k \sin [k(s-l)] \int_{0}^{l} E_{-}\left(s_{0}\right) d s_{0} .
\end{aligned}
$$

Further simplification of (6) leads to

$$
E_{+}(s)=A \cos [k(s-l)+\phi] .
$$

Here

$$
A=\sqrt{\left(E_{-}(l)\right)^{2}+k^{2}\left(\int_{0}^{l} E_{-}\left(s_{0}\right) d s_{0}\right)^{2}}
$$

is the amplitude and $\phi$ is a phase.

The transformer ratio is the ratio of the absolute value of the maximum field behind the bunch to the absolute value of the maximum field inside the bunch, i.e.

$$
R=\frac{\max \left|E_{+}\right|}{\max \left|E_{-}\right|}
$$

Therefore, using Eq. (8) one obtains

$$
R=\sqrt{\left(\frac{E_{-}(l)}{\max \left|E_{-}\right|}\right)^{2}+k^{2}\left(\int_{0}^{l} \frac{E_{-}\left(s_{0}\right)}{\max \left|E_{-}\right|} d s_{0}\right)^{2}} .
$$

We assume that $E_{-}(s)<0$ for $0<s \leq l$ and $E_{-}(s)$ is continuous and bounded on the interval $[0, l]$, then the inequality

$$
\frac{\left|E_{-}(s)\right|}{\max \left|E_{-}(s)\right|} \leq 1
$$

fulfills. Under our assumptions the integral under the square root in (10) can be estimated as

$$
\left(\int_{0}^{l} \frac{E_{-}\left(s_{0}\right)}{\max \left|E_{-}\right|} d s_{0}\right)^{2} \leq l^{2},
$$

and the following inequality fulfills

$$
\left(\frac{E_{-}(l)}{\max \left|E_{-}\right|}\right)^{2} \leq 1 .
$$

Thus, one obtains for the transformer ratio

$$
R \leq \sqrt{1+k^{2} l^{2}}
$$

Among all possible electron bunch distributions with equal length only the distributions that produce almost a constant $E_{-}(s)$ along the electron bunch have the highest transformer ratio

$$
R_{u} \approx \sqrt{1+k^{2} l^{2}} .
$$

The approximation sign here means that the limit cannot be reached in practice. Indeed, if $E_{-}(s)$ is a constant with a jump at the point $s=0$, then the first term in (4) is a Dirac's delta function that is not possible for any practical charge density distribution.

\section{UPPER LIMIT FOR THE ACCELERATING GRADIENT}

Now we define the maximum value of the wake potential per unit length behind the electron bunch as:

$$
\max \left|W_{\|}\right|=\frac{\max \left|E_{+}\right|}{|Q|} .
$$

Using Eqs. (1) and (4), we write for $Q$

$$
Q=\frac{1}{2 \kappa_{\|}}\left[E_{-}(l)+k^{2} \int_{0}^{l} d y \int_{0}^{y} E_{-}(x) d x\right]
$$

and assuming $E_{-}(s)<0$ for $0<s \leq l$ obtain from Eq. (8): 
$\max \left|W_{\|}\right|=2 \kappa_{\|} \frac{\sqrt{\left|E_{-}(l)\right|^{2}+k^{2}\left(\int_{0}^{l}\left|E_{-}\left(s_{0}\right)\right| d s_{0}\right)^{2}}}{\left|E_{-}(l)\right|+k^{2} \int_{0}^{l} d y \int_{0}^{y}\left|E_{-}(x)\right| d x}$

Now we divide numerator and denominator in (18) by $\max \left|E_{-}\right|$and consider:

$$
\begin{aligned}
I_{1} & =\left(\int_{0}^{l} \frac{\left|E_{-}\left(s_{0}\right)\right|}{\max \left|E_{-}\right|} d s_{0}\right)^{2} \\
& =\int_{0}^{l} \int_{0}^{l} \frac{\left|E_{-}(x)\right|}{\max \left|E_{-}\right|} \frac{\left|E_{-}(y)\right|}{\max \left|E_{-}\right|} d x d y
\end{aligned}
$$

and also consider:

$$
I_{2}=\int_{0}^{l} \int_{0}^{y} \frac{\left|E_{-}(x)\right|}{\max \left|E_{-}\right|} d x d y
$$

Then, based on the assumption (11), we have:

$$
I_{2} \geq \int_{0}^{l} \int_{0}^{y} \frac{\left|E_{-}(x)\right|}{\max \left|E_{-}\right|} \frac{\left|E_{-}(y)\right|}{\max \left|E_{-}\right|} d x d y
$$

Evaluating integral $I_{1}$ as:

$$
\begin{aligned}
& \int_{0}^{l} \int_{0}^{l} \frac{\left|E_{-}(x)\right|}{\max \left|E_{-}\right|} \frac{\left|E_{-}(y)\right|}{\max \left|E_{-}\right|} d x d y \\
& =\int_{0}^{l} \int_{0}^{y} \frac{\left|E_{-}(x)\right|}{\max \left|E_{-}\right|} \frac{\left|E_{-}(y)\right|}{\max \left|E_{-}\right|} d x d y \\
& \quad+\int_{0}^{l} \int_{y}^{l} \frac{\left|E_{-}(x)\right|}{\max \left|E_{-}\right|} \frac{\left|E_{-}(y)\right|}{\max \left|E_{-}\right|} d x d y,
\end{aligned}
$$

and changing the order of integration in the second integral, we arrive at

$$
I_{1}=2 \int_{0}^{l} \int_{0}^{y} \frac{\left|E_{-}(x)\right|}{\max \left|E_{-}\right|} \frac{\left|E_{-}(y)\right|}{\max \left|E_{-}\right|} d x d y .
$$

Comparing (21) with (23) we get

$$
\frac{I_{2}}{I_{1}} \geq \frac{1}{2}
$$

Substitution of (19) and (24) into (18) gives

$$
\max \left|W_{\|}\right| \leq 2 \kappa_{\|} \frac{\sqrt{\left(\frac{\left|E_{-}(l)\right|}{\max \left|E_{-}\right|}\right)^{2}+k^{2} I_{1}}}{\frac{\left|E_{-}(l)\right|}{\max \left|E_{-}\right|}+\frac{1}{2} k^{2} I_{1}} .
$$

Because $\frac{\left|E_{-}(l)\right|}{\max \left|E_{-}\right|} \leq 1$, making a smaller denominator in (25) with substitution $\frac{\left|E_{-}(l)\right|}{\max \left|E_{-}\right|} \Rightarrow \frac{1}{2}\left(\frac{\left|E_{-}(l)\right|}{\max \mid E_{-}}\right)^{2}$ only increases the right part in (25) and consequently the inequality

$$
\max \left|W_{\|}\right| \leq 2 \kappa_{\|} \frac{\sqrt{\left(\frac{\left|E_{-}(l)\right|}{\max \mid E_{-}}\right)^{2}+k^{2} I_{1}}}{\frac{1}{2}\left(\frac{\left|E_{-}(l)\right|}{\max \left|E_{-}\right|}\right)^{2}+\frac{1}{2} k^{2} I_{1}}
$$

holds. With this change we can now relate the upper limit for $\max \left|W_{\|}\right|$with the transformer ratio defined in (10) as:

$$
\max \left|W_{\|}\right| \leq \frac{4 \kappa_{\|}}{R} .
$$

and write for the maximum accelerating gradient:

$$
\max \left|E_{+}\right| \leq \frac{4 \kappa_{\|}|Q|}{R}
$$

Since $\max \left|W_{\|}\right| \leq 2 \kappa_{\|}$, the inequalities (27) and (28) are applicable only for $R \geq 2$.

A more accurate definition of the upper limit for the accelerating gradient can be obtained in many special cases when $\frac{\left|E_{-}(l)\right|}{\max \left|E_{-}\right|}=1$. From (25) using Eqs. (10) and (19) one can obtain

$$
\max \left|W_{\|}\right| \leq 2 \kappa_{\|} \frac{2 R}{1+R^{2}},
$$

and finally for the upper limit of the accelerating gradient

$$
\max \left|E_{+}\right| \leq 2 \kappa_{\|}|Q| \frac{2 R}{1+R^{2}} .
$$

The equal sign in (30) is realized in the most desirable case of the constant longitudinal field inside the electron bunch.

The result shown by Eqs. (28) and (30) has a simple physics meaning. Accelerating field amplitude $\max \left|E_{+}\right|$ begins to drop when $R$ increases. According to (15) this happens when the electron bunch begins to occupy a significant fraction of the wavelength $\lambda$ in which case the cooperative interference of the radiation fields of the individual electrons begins to become looser and the destructive interference between the head and tail electrons begins to emerge. The intended use of the destructive interference is suppression of the decelerating field inside the electron bunch and the accelerating field behind the bunch, but with carefully manipulated imbalance (controlled by the charge distribution in the electron bunch) yielding high transformer ratio. Since large transformer ratio is needed to boost the efficiency of the accelerator measured in terms of the energy transfer from the drive bunch to the witness bunch, we have proven that this can only be done at the expense of a reduced energy gain for the witness bunch per a unit length and, thus, at the expense of having a longer accelerator. This conclusion applies to any type of the wakefield accelerator with the single mode Green's function and independent of the environment used to create the wakefield. 


\section{MULTIMODE CASE}

Here we consider the CWA with the wakefield producing environment characterized by the Green's function that has more than one mode.

Let $k_{n}$ be the wave vector and $\kappa_{n}$ be the loss factor for the $n$th mode. Let $\tilde{q}(k)$ be the Fourier transform of $q(s)$. Consequently,

$$
\kappa_{\|}=\sum_{n=1}^{\infty} \kappa_{n}
$$

and

$$
\max \left|E_{+}^{\mathrm{ml}}\right| \leq 2 \sum_{n=1}^{\infty} \kappa_{n}\left|\tilde{q}\left(k_{n}\right)\right|
$$

Here we denote $E_{+}^{\mathrm{ml}}$ as a longitudinal field behind the bunch in the multimode case.

Because of the bunching factor, we assume that $\tilde{q}(k)$ overlaps only $N$ lowest modes and that there is such a mode $m$ that $\left|\tilde{q}\left(k_{m}\right)\right|>\left|\tilde{q}\left(k_{n}\right)\right|$ for all $n$ from 1 to $N$ and $n \neq m$. In this case

$$
\sum_{n=1}^{N} \kappa_{n}\left|\tilde{q}\left(k_{n}\right)\right| \leq\left|\tilde{q}\left(k_{m}\right)\right| \sum_{n=1}^{N} \kappa_{n}
$$

and using Eqs. (31) and (32) one can write:

$$
\max \left|E_{+}^{\mathrm{ml}}\right| \leq 2 \kappa_{\|}\left|\tilde{q}\left(k_{m}\right)\right| .
$$

Since $\max \left|E_{+}\right|$in the single mode case equals to $2 \kappa_{\|}\left|\tilde{q}\left(k_{m}\right)\right|$, then we are able to prove, under the assumption given above, that the collinear wake field accelerators with the multimode Green's function cannot have higher accelerating gradient than that in the CWA with the single mode Green's function.

\section{ILLUSTRATIONS}

We illustrate the results obtained in the previous sections for a single mode Green's function using two charge density distributions in the electron bunch shown in Fig. 1. The case (a) has a characteristic triangular shaped
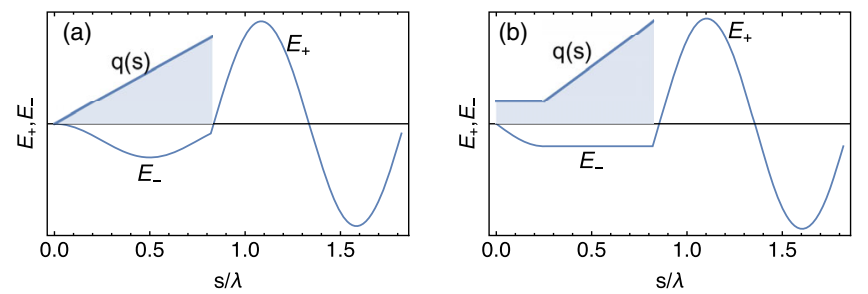

FIG. 1. Electron density distribution and normalized wake field. See text for explanation of the specific plots.

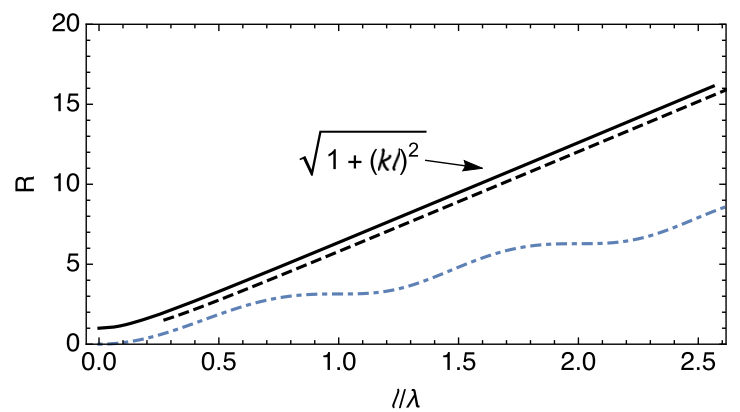

FIG. 2. Transformer ratio. Solid black line is given by Eq. (15). The dot dashed and dashed curves correspond to the cases (a) and (b) described in the text. As expected, the dashed curve lies only slightly below the theoretical maximum.

distribution with a linear growth from $s=0$ to the end of the bunch at $s=l$ previously considered in a number of publications (see, i.e., $[9,10])$. In this case $E_{-}$is not constant along the electron bunch length, which makes obtaining high $R$ (and high max $\left|E_{+}\right|$for a large $R$ ) more difficult (see, Figs. 2 and 3). Another disadvantage of a nonconstant $E_{-}$is the loss of the efficiency in the energy transfer from the drive bunch to the witness bunch in the collinear wake field accelerator since the acceleration will basically end when electrons with the highest rate of energy losses give up their entire energy while other electrons still have some energy left. In the case of a constant $E_{-}$all electrons lose their energies with the same rate.

The "door step" electron density distribution $q(s)=$ $1+\theta[k s-\pi / 2](k s-\pi / 2)$ proposed in [9] and used in the case (b) largely solves this problem, ensuring an extended range of the constant $E_{-}$along the bunch length. Therefore the attainable $R$ and $\max \left|E_{+}\right|$are not much different from maximum theoretical values as seen in Figs. 2 and 3.

The next two examples use the multimode Green's function and demonstrate that indeed the upper limits for the transformer ratio and for the accelerating gradient defined in Sec. II and Sec. III are not exceeded.

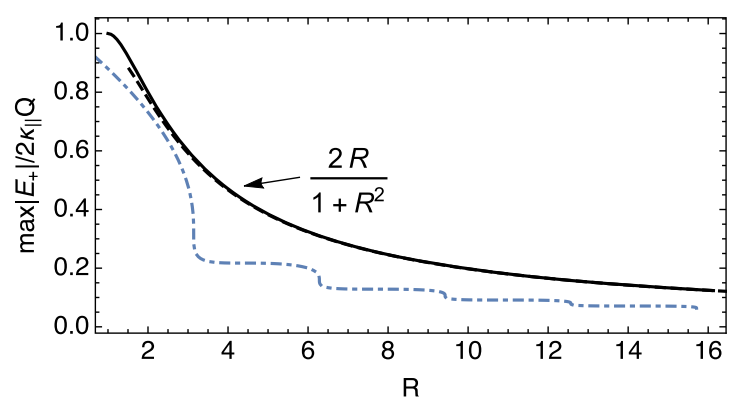

FIG. 3. The normalized maximum accelerating field as a function of the transformer ratio. The solid black line is given by Eq. (30). The dot dashed and dashed lines correspond to the cases (a) and (b) described in the text. As seen in this plot, the case (b) is practically indistinguishable from the theoretical maximum. 


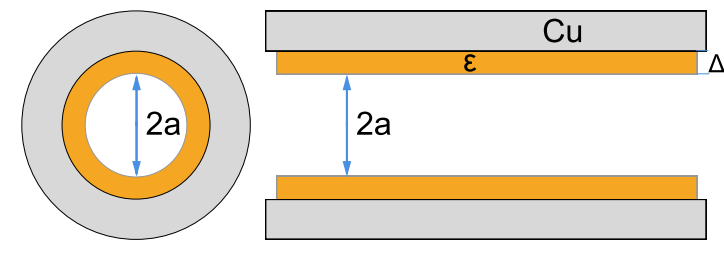

FIG. 4. Schematics of the dielectric lined waveguide.
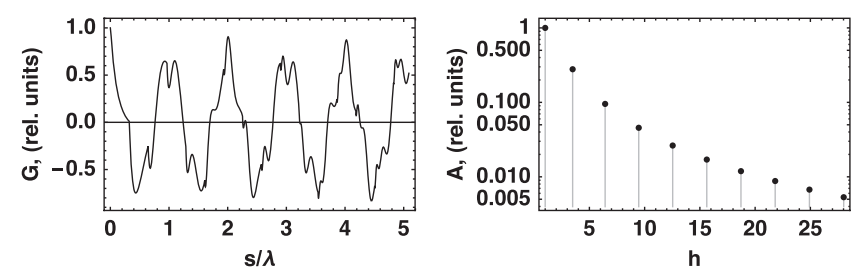

FIG. 5. Green's function (left panel) and spectrum (right panel) normalized on the maximum values for the case of a dielectric lined copper tube with $\Delta=200 \mu \mathrm{m}$. Here $\mathrm{h}$ is the harmonic number, $\lambda$ is the wavelength of the harmonic with the maximum amplitude.

In the first multimode example we consider a dielectric lined copper tube with the inner radius $a=1 \mathrm{~mm}$ and a thickness of the dielectric layer $\Delta=200 \mu \mathrm{m}$ with the dielectric constant $\epsilon=3.5$ (see Fig. 4). Figure 5 shows the Green's function and its spectrum for this case, calculated using formulas taken from Ref. [17] and benchmarked with CST [18]. The charge density distribution for this case was meticulously prepared to yield a quasiconstant $E_{-}$using the algorithm described in the Appendix. This distribution (shown in Fig. 7(c) and continuously scaled with the changing value of $l$ ) was used to calculate $R$ as a function $l / \lambda$ and $\max \left|E_{+}\right|$as a function of $R$. Here $\lambda$ is the wavelength of the first mode. The result is shown in Figs. 8 and 9 where we also show the upper values calculated using Eqs. (15) and (30).

In the second example we consider the dielectric lined copper tube with the same $a=1 \mathrm{~mm}$, but with the $4 \mathrm{~mm}$ dielectric layer thickness. It allowed us to obtain the Green's function with the spectrum where the third mode is the dominant one as seen in Fig. 6. As in the two cases
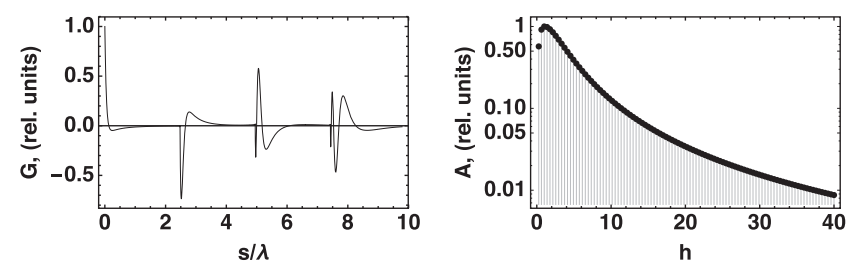

FIG. 6. Green's function (left panel) and spectrum (right panel) normalized on the maximum values for the case of a dielectric lined copper tube with $\Delta=4 \mathrm{~mm}$. Here $\mathrm{h}$ is the harmonic number, $\lambda$ is the wavelength of the harmonic with the maximum amplitude.
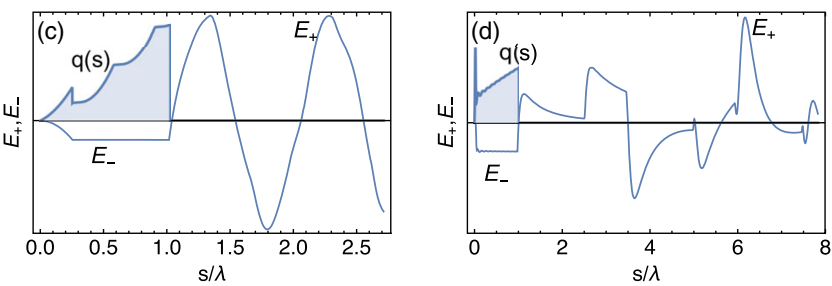

FIG. 7. Electron density distribution and normalized wakefield. See text for explanation of the specific plots.

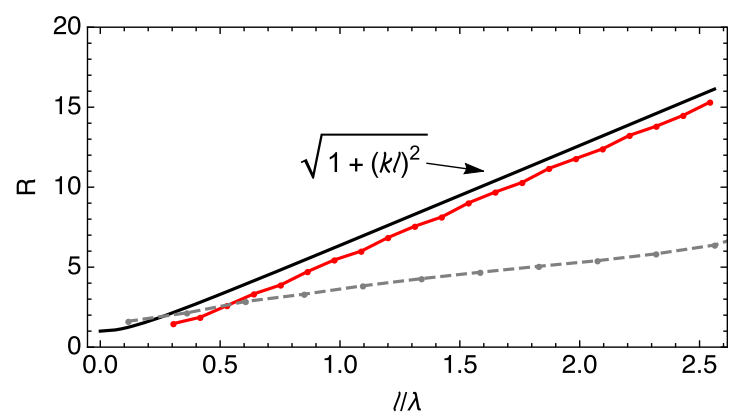

FIG. 8. Transformer ratio. Solid black line is the same as in Fig. 2. The dot-solid and dot-dashed lines correspond to cases (c) and (d) in Fig. 7.

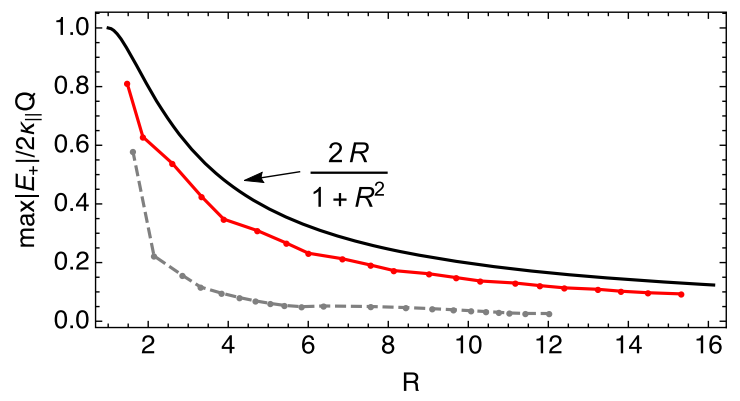

FIG. 9. The normalized maximum accelerating field as a function of the transformer ratio. The solid black line is the same as in Fig. 2. The dot-solid and dot-dashed lines correspond to cases (c) and (d) in Fig. 7.

mentioned above, the special charge density distribution was meticulously prepared to yield a quasi-constant $E_{-}$. This distribution [shown in Fig. 7(d)] was used to calculate $R$ and $\max \left|E_{+}\right|$using the wavelength of the 3rd mode. The result is shown in Figs. 8 and 9. A dramatic fall of $\max \left|E_{+}\right|$can be attributed to a quick decoherence of the radiation fields of the individual electrons with the increased bunch length.

\section{CONCLUSION}

It has been shown that the transformer ratio $R$ and the maximum energy gain given by the accelerating field $\max \left|E_{+}\right|$in the collinear wake field accelerator should not be treated independently. In fact, their mutual 
relationship is tightly constrained to $\max \left|E_{+}\right| \propto 1 / R$ when large $R$ values are used for obtaining the efficient energy transfer from the drive bunch to the witness bunch. It has been also shown that the best result in obtaining the highest efficiency for a given energy gain is achieved when the electron bunch distribution is meticulously shaped (in concord with a consideration of the specific wake field producing environment with its specific wake field Green's function) to yield a quasiconstant decelerating field $E_{-}$along the bunch length. The two results summarized here are applicable to the collinear wake field accelerators driven by a single bunch and not applicable to ones that use more than one drive bunch.

Another important parameter to be considered is the magnitude of the accelerating field which is strictly defined by the product of the total bunch charge and the loss factor, i.e., $Q \kappa_{\|}$(see, e.g., $[19,20]$ ). Evidently, these are not free parameters because the limitations on both are given by considerations of stability of the drive bunch propagating the wake field creating environment and gradually decelerating (possible tradeoffs are discussed for example in [21]).

\section{ACKNOWLEDGMENTS}

This work was supported by the U.S. Department of Energy, Office of Science, Office of Basic Energy Sciences, under Contract No. DE-AC02-06CH11357; by the U.S. National Science Foundation under Award No. PHY1549132, the Center for Bright Beams and under Award No. PHY-1535639.

\section{APPENDIX: OPTIMIZATION OF THE BUNCH SHAPE TO OBTAIN SPECIFIC $E_{-}$}

Let us consider Eq. (2) for an arbitrary Green's function

$$
E_{-}(s)=\int_{0}^{s} G\left(s-s_{0}\right) q\left(s_{0}\right) d s_{0}, \quad s \leq l .
$$

If $E_{-}(s)$ is known and satisfies the condition $E_{-}(0)=0$, we can calculate $q$ treating (A1) as a Volterra equation as follows [16]:

$$
q(s)=\frac{E_{-}^{\prime}(s)}{2 \kappa_{\|}}+\int_{0}^{s} \frac{K\left(s-s_{0}\right) E_{-}^{\prime}\left(s_{0}\right)}{2 \kappa_{\|}} d s_{0}
$$

with a resolvent kernel given by

$$
K(x)=\mathfrak{Q}^{-1}\left[\frac{1}{p \tilde{G}(p)}-1\right]
$$

where

$$
\tilde{G}(p)=\frac{\mathfrak{Q}[G(x)]}{2 \kappa_{\|}} .
$$

Here $\mathfrak{L}$ and $\mathfrak{Q}^{-1}$ are the operators of direct and inverse Laplace transforms, given by

$$
\begin{aligned}
\tilde{f}(p) & =\mathfrak{Q}[f(x)]=\int_{0}^{\infty} e^{-p x} f(x) d x, \\
g(x) & =\mathfrak{Q}^{-1}[\tilde{g}(p)]=\frac{1}{2 \pi i} \int_{c-i \infty}^{c+i \infty} e^{p x} \tilde{g}(p) d p .
\end{aligned}
$$

Now defining the electron density distribution as

$$
\rho(s)=\frac{q(s)}{Q}
$$

and using (A2) and (1) we arrive at

$$
\rho(s)=\frac{E_{-}^{\prime}(s)+\int_{0}^{s} K\left(s-s_{0}\right) E_{-}^{\prime}\left(s_{0}\right) d s_{0}}{E_{-}(l)+\int_{0}^{l} \int_{0}^{s} K\left(s-s_{0}\right) E_{-}^{\prime}\left(s_{0}\right) d s_{0} d s} .
$$

For the Green's function with a finite amount of modes Eq. (A3) can be evaluated analytically, in which case substitution of $K(x)$ into Eq. (A7) allows one to calculate $\rho(s)$ for any desired $E_{-}(s)$, in particular obtaining the unique $\rho(s)$ correspondent to cases with the constant longitudinal field inside the electron bunch.

Here we demonstrate it for two cases, each having:

$$
E_{-}(s)=E_{0} \theta(s)
$$

and thus possessing:

$$
\rho(s)=\frac{\delta(s)+K(s)}{1+\int_{0}^{l} K(s) d s}
$$

as follows from (A7), where $\delta(s)$ is the Dirac's delta function.

In the first case we consider a single mode Green's function $G(s)=2 \kappa_{\|} \cos (k s)$. Using (A4) and (A3) we obtain:

$$
\tilde{G}(p)=\frac{p}{k^{2}+p^{2}}, \quad \tilde{K}(p)=\frac{k^{2}}{p^{2}}, \quad K(s)=k^{2} s
$$

and arrive using (A9) at a well known result of a triangular shaped electron density distribution with the spike at $s=0$, i.e.:

$$
\rho(s)=\frac{\delta(s)+k^{2} s}{1+1 / 2 k^{2} l^{2}} .
$$

In the second case we consider the Green's function with two modes and with the first mode dominating the second, i.e.:

$$
G(s)=2 \kappa_{\|} \frac{\cos \left(k_{1} s\right)+\alpha \cos \left(k_{2} s\right)}{1+\alpha},
$$


with $\alpha<1$. Now obtaining from (A4):

$$
\tilde{G}(p)=\frac{1}{1+\alpha}\left[\frac{p}{k_{1}^{2}+p^{2}}+\frac{\alpha p}{k_{2}^{2}+p^{2}}\right]
$$

we have for the resolvent kernel:

$$
\begin{aligned}
K(s)= & \frac{(1+\alpha) k_{1}^{2} k_{2}^{2}}{k_{2}^{2}+\alpha k_{1}^{2}} s \\
& +\frac{\alpha\left(k_{2}^{2}-k_{1}^{2}\right)^{2} \sin \left(s \sqrt{\frac{k_{2}^{2}+\alpha k_{1}^{2}}{1+\alpha}}\right)}{\sqrt{1+\alpha}\left(k_{2}^{2}+\alpha k_{1}^{2}\right)^{3 / 2}} .
\end{aligned}
$$

Substituting (A14) into (A9) and omitting for brevity the normalization coefficient we arrive at

$$
\begin{aligned}
\rho(s) \propto & \delta(s)+\frac{(1+\alpha) k_{1}^{2} k_{2}^{2}}{k_{2}^{2}+\alpha k_{1}^{2}} s \\
& +\frac{\alpha\left(k_{2}^{2}-k_{1}^{2}\right)^{2} \sin \left(s \sqrt{\frac{k_{2}^{2}+\alpha k_{1}^{2}}{1+\alpha}}\right)}{\sqrt{1+\alpha}\left(k_{2}^{2}+\alpha k_{1}^{2}\right)^{3 / 2}} .
\end{aligned}
$$

We note that the third term in (A15) is not necessarily small even if $\alpha$ is small. Therefore, comparing (A15) to (A11) leads us to a conclusion that there must always be a unique electron density distribution matching each specific Green's function that allows for maintaining the constant longitudinal field inside the electron bunch.

[1] G.-A. Voss and T. Weiland, DESY Report No. 82-074, 1982.

[2] R. J. Briggs, T. J. Fessenden, and V. K. Neil, Electron acceleration, in Proc. 9th Int. Conf. High Energy Accel. (SLAC-R-839, Stanford, USA, 1974), p. 278.

[3] M. Friedman, Autoacceleration of an Intense Relativistic Electron Beam, Phys. Rev. Lett. 31, 1107 (1973).

[4] E. A. Perevedentsev and A. N. Skrinsky, Using intense proton beams of large proton accelerators to drive wake fields in linear accelerators, (in Russian), Proc. 6th AllUnion Conf Charged Part. Accel., Dubna (1978), Vol. 2, p. 272.

[5] A. M. Sessler, The free electron laser as a power source for a high-gradient accelerating structure, AIP Proc. 91, 154 (1982).
[6] P. Chen, R. W. Huffad, and J. M. Dawson, Bull. Am. Phys. Soc. 29, 1355 (1984).

[7] Y. Chin, The wake field acceleration using a cavity of elliptical cross section, in Proc. Lin. Accef. Conf. (LINAC '84), Seeheim/Darmstadt (1984), p. 159.

[8] W. Gai, P. Schoessow, B. Cole, R. Konecny, J. Norem, J. Rosenzweig, and J. Simpson, Experimental Demonstration of Wake-Field Effects in Dielectric Structures, Phys. Rev. Lett., 61, 2756 (1988),

[9] K. L. F. Bane, P. Chen, and P. W. Wilson, On collinear wake field acceleration, IEEE Trans. Nucl. Sci. 32, 3524 (1985).

[10] B. Jiang, C. Jing, P. Schoessow, J. Power, and W. Gai, Formation of a novel shaped bunch to enhance transformer ratio in collinear wakefield accelerators, Phys. Rev. ST Accel. Beams 15, 011301 (2012).

[11] J. Power, Presentation at 2011 Argonne Workshop on Dielectric Wake Field Accelerator, 2011 (unpublished).

[12] F. Lemery and P. Piot, Tailored electron bunches with smooth current profiles for enhanced transformer ratios in beam-driven acceleration, Phys. Rev. ST Accel. Beams 18, 081301 (2015).

[13] V. M. Tsakanov, On collinear wake field acceleration with high transformer ratio, Nucl. Instrum. Methods Phys. Res., Sect. A 432, 202 (1999).

[14] B. Zotter and S. Kheifets, Impedances and Wakes in High Energy Particle Accelerators (World Scientific Publishing Company, Singapore, 1998).

[15] A. Chao, in Physics of Collective Beam Instabilities in High Energy Accelerators (Wiley and Sons, New York, 1993).

[16] A. D. Polyanin and A. V. Manzhirov, Handbook of Integral Equations (CRC Press, Boca Raton, 1998).

[17] A. M. Altmark and A. D. Kanareykin, Annular cherenkov high gradient wakefield accelerator: beam-breakup analysis and energy transfer efficiency, J. Phys. Conf. Ser. 357, 012001 (2012).

[18] http://www.cst.com.

[19] S. S. Baturin and A. D. Kanareykin, Cherenkov radiation from short relativistic bunches: General approach, Phys. Rev. Lett. 113, 214801 (2014).

[20] S.S. Baturin and A. D. Kanareykin, New method of calculating the wakefields of a point charge in a waveguide of arbitrary cross section, Phys. Rev. Accel. Beams 19, 051001 (2016).

[21] C. Li, W. Gai, C. Jing, J. G. Power, C. X. Tang, and A. Zholents, High gradient limits due to single bunch beam breakup in a collinear dielectric wakefield accelerator, Phys. Rev. ST Accel. Beams 17, 091302 (2014). 